\title{
AVENIDAS FLUVIALES Y PRECIPITACIONES EN LAS CUENCAS DE LOS RÍOS JARAMA-TAJO AL FINAL DEL SIGLO XVI
}

\author{
Teresa Bullón Mata \\ Departamento de Geografía. Universidad Autónoma de Madrid \\ teresa.bullon@uam.es
}

\section{RESUMEN}

En este trabajo se presentan los resultados de una reconstrucción histórica realizada a partir de fuentes documentales de las precipitaciones y avenidas fluviales en los últimos cincuenta años del siglo XVI en el sector noroccidental de la cuenca del Tajo. Los datos cualitativos se han analizado mediante la traducción cuantitativa de los mismos a índices numéricos. Se diferencian tres conjuntos de años con significados hidrológicos diferentes. El primero, desde 1557 a 1575, tiene precipitaciones regulares, que provocan avenidas de menor intensidad. El segundo, desde 1576 a 1584, se caracteriza por precipitaciones y avenidas escasas. El tercero, desde 1585 a 1599, tiene precipitaciones intensas, con avenidas de gran importancia, intercaladas entre grandes sequías.

Palabras clave: Climatología histórica, cuenca del Tajo, avenidas fluviales, precipitaciones, sequías.

\section{ABSTRACT}

This work is about the results of a historical reconstruction realized from documentary sources of rainfalls and floods that have been detected in the last fifty years of the 16th century in the north - western part of the Tagus basin. Qualitative data have been analyzed by means of their quantitative translation to numerical indexes. Three sets of years with hydrological different meanings are found. The first one, from 1557 to 1575 , has regular rainfalls and floods of minor intensity. The second one, from 1576 to 1584 , is characterized

Fecha de recepción: enero 2011.

Fecha de aceptación: julio 2012. 
by rainfalls and scanty floods. The third party, from 1585 to 1599 , has intense rainfalls, with floods of great importance, alternating with severe droughts.

Key words: Historical climatology, Tajo basin, floods, precipitations, droughts.

\section{INTRODUCCIÓN}

En este trabajo se presentan los resultados de una reconstrucción histórica realizada con de fuentes documentales sobre las relaciones entre precipitaciones y avenidas del área de Madrid (Figura 1), situada en el centro de la Península Ibérica.

El sector geográfico estudiado tiene un carácter mediterráneo continental, en el que se desarrollan a distintas altitudes diferentes pisos bioclimáticos mediterráneos. El marco climático regional se define por una amplia variación térmica anual, precipitaciones equinociales y sequía entre junio y septiembre. Las escorrentías se canalizan a través de las grandes cuencas fluviales del Jarama y Tajo. Una gran parte del área de estudio está constituido encinares muy transformados por la acción humana, que alternan con pastizales extensivos, cultivos y formaciones de matorrales esteparios desarrollados a veces sobre sustratos de yeso.

Los ríos que drenan el sector indicado tienen sus cabeceras en las Sierras de Guadarrama y Somosierra, a altitudes superiores a los 2000 m, en las que existe en la actualidad una intensa acumulación nival invernal, así como actividad periglaciar, asociada a numerosos ciclos de hielo-deshielo. Durante el Pleistoceno estas cabeceras alojaron circos glaciares. Desde la montaña la red fluvial se extiende por los relieves llanos de la cuenca sedimentaria de Madrid, de unos $500 \mathrm{~m}$. de altitud media, en los que las condiciones de aridez se incrementan hacia el sur, ya que la precipitación media anual a $1890 \mathrm{~m}$. (Observatorio Puerto de Navacerrada) es de $1511 \mathrm{~mm} / \mathrm{año} \mathrm{y}$ en el extremo del área de estudio, situado a unos $120 \mathrm{~km}$ al SE, (Observatorio de Aranjuez) es de 448 mm/año.

Las llanuras aluviales que forman los ríos han sido históricamente espacios arbolados con dedicación parcial a pastos, caza y cultivos. A partir de la segunda mitad del siglo XX este espacio se dedica a la extracción de áridos, lo que produce una degradación muy intensa de los cauces y sus riberas. En el momento actual, la dinámica fluvial está muy alterada y se ha producido el afloramiento en superficie de los niveles freáticos, cuyas aguas se encuentran estancadas en numerosas lagunas ( Molina y Berrocal 2006, Gallego y Sánchez 2006 , Alonso y Garzón, 1997, Vizcaíno y otros 2003).

La investigación realizada se asocia con el estudio de la variabilidad climática en las tierras de interior del borde meridional europeo y con el análisis de las condiciones climáticas propias del siglo XVI, período del que hay muy poca información climática o medioambiental detallada. Por último, se aportan numerosos datos inéditos sobre avenidas fluviales. Por todo ello, la investigación realizada mejora el conocimiento de los sistemas fluviales en su régimen natural mucho antes de que existieran registros de aforo y contribuye a completar las secuencias históricas necesarias para la prevención de las avenidas, en línea con lo expresado en la directiva europea 903/60 relativa a la evaluación y gestión de los riesgos de inundación.

Se utilizan fuentes documentales escritas o gráficas, que permiten conocer de modo directo la influencia que tuvieron los diferentes acontecimientos hidrológicos y meteorológi- 


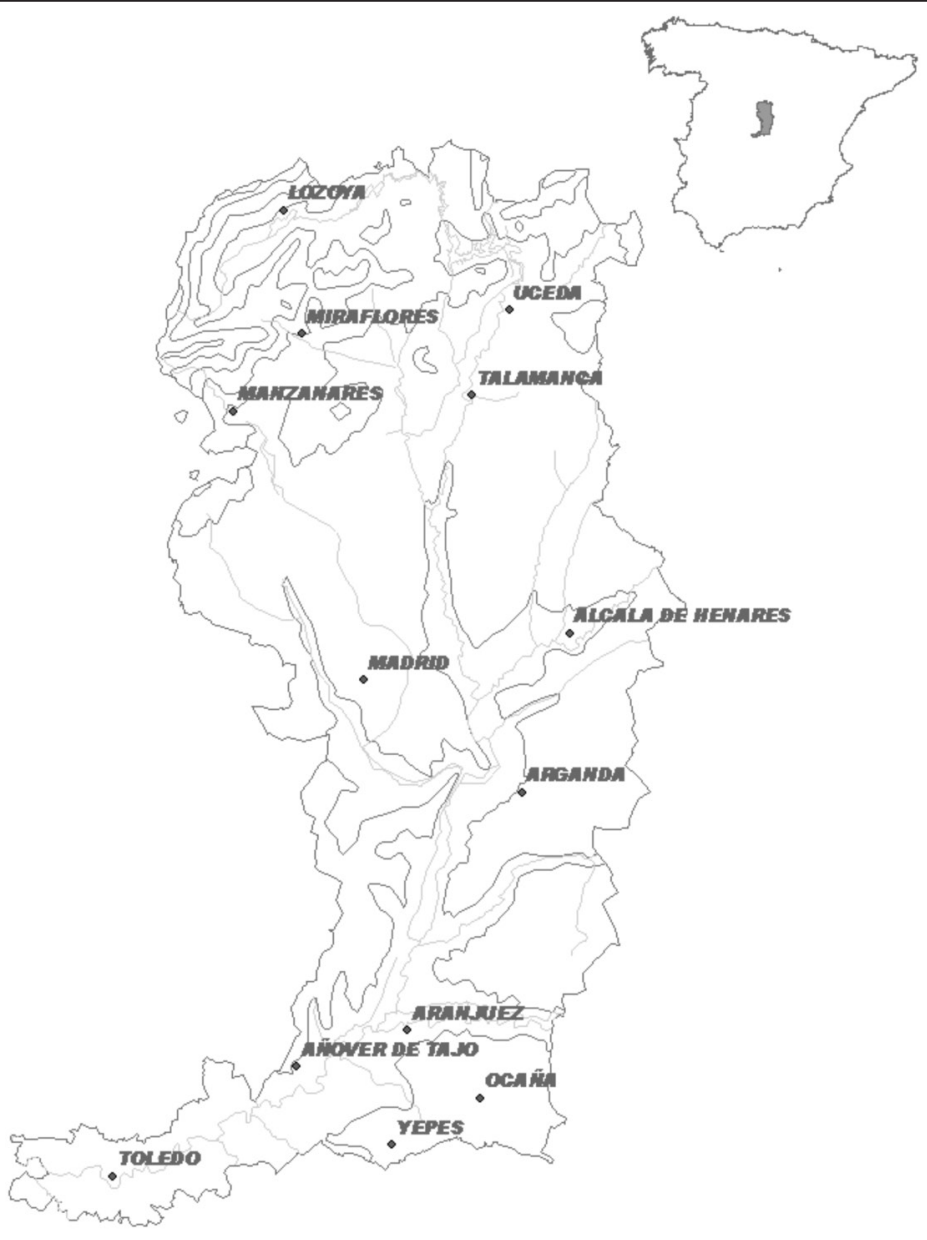

Fuente: elaboración propia a partir de la cartografía digital de la CAM.

cos en las personas, bienes y servicios y que facilitan la determinación de la intensidad, magnitud y frecuencia de los referidos acontecimientos mediante la transformación de los datos cualitativos en índices numéricos. Los archivos consultados están ubicados en el Archivo General de Palacio, el Archivo General de Simancas, Archivo Histórico Nacional (sección de Nobleza), Archivos municipales de las villas de Madrid y Torrelaguna, así como secciones de manuscritos de la Biblioteca Nacional de España, y de la British Library. 


\section{OBJETIVOS Y MÉTODOS DE TRABAJO}

Los objetivos concretos propuestos en este trabajo son: 1 Determinar los diferentes tipos de avenidas que se describen en los documentos analizados. 2 Definir las características principales de la evolución climática del final del siglo XVI a partir del estudio de las fases de precipitación y avenidas que se han detectado. 3 Comprobar el funcionamiento del complejo natural y humano organizado en torno a la dinámica fluvial de los ríos.

El trabajo consiste en la obtención del significado climático e hidrológico de los datos documentales y se realiza en tres fases: interpretación de la información cualitativa, cuantificación de los datos y análisis de los mismos mediante métodos estadísticos o cualitativos.

\section{La interpretación de los datos documentales}

La información directa obtenida de las diferentes fuentes consultadas se ha realizado mediante la lectura de los datos recogidos sobre los textos originales literales. La interpretación de los datos recogidos depende de: 1 La asignación de la fecha de ocurrencia, pues, aunque habitualmente los documentos están fechados con día, mes y año, los acontecimientos explicados en ellos pueden referirse a hechos acaecidos en otros momentos. 2 Tipo de dato obtenido, que puede ser directo, en el que se describe explícitamente un acontecimiento meteorológico o de avenidas fluviales, a veces con indicación de los daños o consecuencias objetivas de ello, o indirecto a partir del que se tienen los daños o beneficios asociados, sin alusión directa al fenómeno que lo produjo. En la estimación de las sequías y precipitaciones se han tenido en cuenta como datos indirectos el estado de las cosechas, el grado de desarrollo de la hierba y la capacidad de ésta para alimentar al ganado o la abundancia y salud de la caza mayor y menor. En la estimación de las avenidas se han considerado los daños en los puentes, las roturas y deterioros de las presas, canales y acequias, las evidencias de erosiones en la llanura de inundación y las repoblaciones o talas de la vegetación riparia.

\section{Transformación de los datos en índices numéricos}

Se han establecido índices numéricos, que permiten realizar operaciones estadísticas. La transformación en valores numéricos de los datos cualitativos es habitual en los trabajos de climatología o de hidrología históricas: Martin Vide y Barriendos (1995), Barriendos y Coeur (2004); Llasat et al., (2004) Brázdil et al. (2010), Bullón (2010). Las normas que se han seguido responden a estos procedimientos, aunque se han diseñado índices propios, para adaptarlos a las peculiaridades de los datos utilizados.

Los datos cualitativos se convierten en valores numéricos a través de índices de intensidad - duración para precipitaciones y de intensidad - magnitud para avenidas. Cada hecho concreto, que forma parte de una de las series referidas, es valorado según su intensidad, con un rango de tres clases diferentes: $1=$ poca intensidad; $2=$ intensidad moderada, $3=$ intensidad muy importante. La duración se define mediante las categorías: 1= uno o dos días, 2= una o varias semanas, $3=$ una estación o período largo de tiempo. Para valorar la magnitud de avenidas se utiliza una secuencia relativa a la importancia hidrológica de los cauces afectados: $1=$ cabeceras, $2=$ tramos medios, $3=$ tramos regionales. Posteriormente, estos valores 
numéricos son agrupados por meses. El valor de cada mes es el resultado de la suma del $70 \%$ del promedio de intensidad de todos los datos recogidos en ese mes y el $30 \%$ del promedio de duración o importancia del cauce afectado.

\section{Análisis estadístico de los datos}

Los resultados se analizan estadísticamente teniendo en cuenta cada una de las series obtenidas y las relaciones entre ellas. A partir del análisis inicial de las medidas de tendencia central, de variabilidad y de forma mediante las que se describen estadísticamente cada una de las dos series utilizadas, se determina si las muestras proceden de una distribución normal.

La variabilidad temporal a medio y largo plazo se determina gráficamente a partir de las medias móviles de 5 años. También se utiliza la curva de valores normales acumulados, en la cual los tramos descendentes coinciden con períodos de disminución de las de avenidas o de precipitaciones y los ascendentes con aumento de las mismas.

Los modelos predictivos más adecuados para el análisis temporal de las series se obtienen mediante un análisis de los residuos de los posibles modelos a utilizar. En cualquier caso, el test secuencial no paramétrico de Kendall es el que fija las etapas más destacadas en la evolución temporal de las variables.

\section{RESULTADOS}

\section{Datos cualitativos}

\section{La tipología de las crecidas}

Las crecidas estacionales son uno de los rasgos más importancia en la dinámica de este sistema fluvial en los tramos medios y regionales y constituyen el principal motor de funcionamiento de la explotación del territorio. Estas crecidas ocurren dos veces cada año, una en invierno, cuando hay nieve en la sierra, que parecen ser las de mayor caudal, y otra en primavera, entre Marzo y Mayo. A través de estas crecidas las márgenes del río adquieren la humedad necesaria para la fertilización de la tierra, así como para la germinación y el crecimiento vegetal.

Las crecidas extraordinarias pueden producir desperfectos de diversa intensidad, aunque en la valoración posterior el efecto beneficioso de la inundación de las tierras atenúa el balance final de daños. Estas crecidas, causadas por lluvias o fusiones nivales importantes, ocurren en 1556-57, 1585-87 y 1590-91 y 1593-94 dañando puentes, acequias y todo tipo de construcciones o bienes. Las que ocurrieron en la década de los 90 fueron especialmente intensas.

Asimismo se han detectado flash floods, que ocurrieron en meses estivales a consecuencia de precipitaciones cortas e intensas, que dan aumentos súbitos del caudal de agua. Las variedades de éstas últimas oscilan desde las que responden de inmediato a una precipitación intensa: un aguaducho que sobrevino.... que bajó los valles y llevó mucha parte de la acequia que estaba hecha... septiembre1579. (Archivo General de Simancas, Casas y Sitios Reales (AGS C y SR), legajo 254-7/189), a la creciente que se presenta inesperadamente, no 
habiendo llovido nada in situ o habiendo llovido mucho menos de lo que indica el volumen de agua que desciende. La más interesante de todas, por el efecto súbito y diferencias en el estilo de movimiento del agua y de la carga sedimentaria, es la producida en 1570: aquí había llovido mucho el jueves y había crecido el Tajo aquel día y bien se entendió que no podía ser la creciente de lo que el dicho jueves había llovido, aunque fue harto, por venir tan presto....El agua venía tan turbia y tan mala que echó fuera pesca muerta. Vinieron muchas ovejas ahogadas el río abajo y algunas bestias y puercos y madera de pino. 4 de julio de 1570(AGS C y SR legajo 253-5/70).

\section{Las modificaciones del trazado de los canales}

La capacidad de migración del canal habitual en la llanura aluvial es muy grande. Los rasguños y mapas conservados de algunos tramos indican que tienden a mantenerse los antiguos límites de campos o propiedades que estaban delimitados por canales activos (madres), una vez que fueron abandonados (Figura 2). Los numerosos procesos legales que se establecen por la determinación de los límites de las propiedades y por la asignación de las nuevas tierras abandonadas por los ríos, reflejan los importantes problemas que se generaron a consecuencia de esta dinámica fluvial y la gran incertidumbre sobre la conservación de las propiedades, que afectaba a los dueños y arrendatarios de los terrenos. Los tramos fluviales de mayor riesgo de cambio son los situados en áreas próximas a las confluencias de los tributarios de mayor importancia con el río principal.

Figura 2

EL RIOO JARAMA EN EL SOTO DE EL PIUL EN EL SIGLO XVII

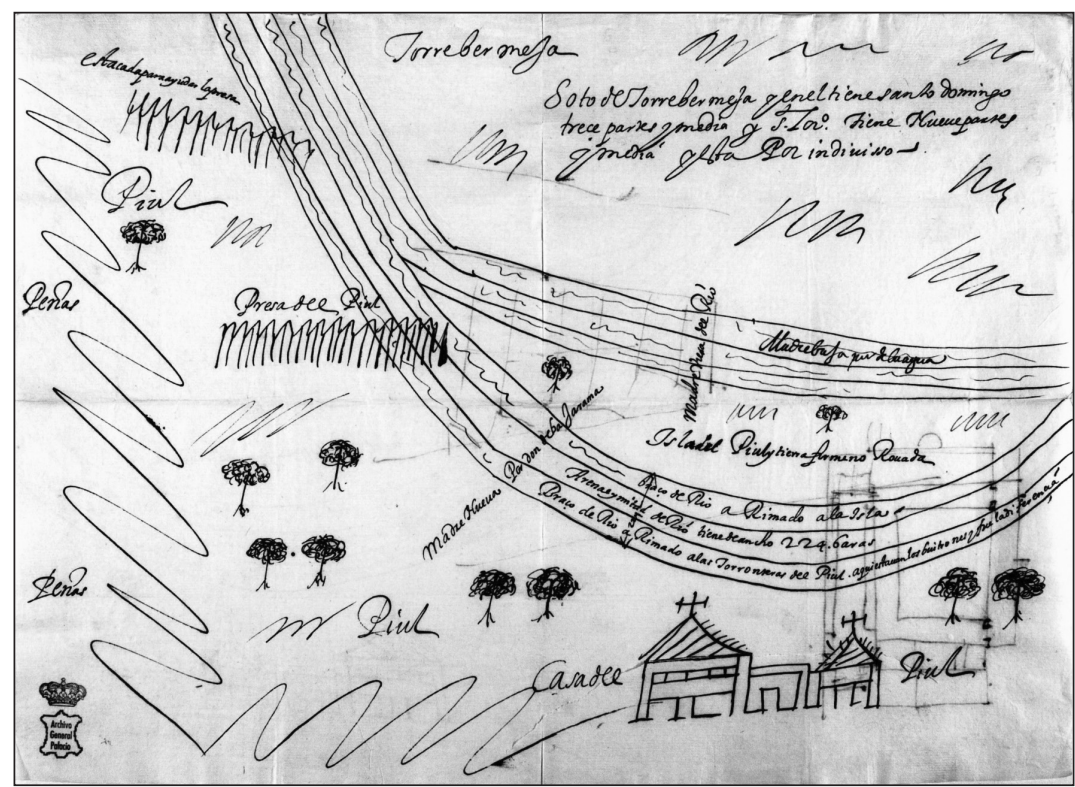

Fuente: Archivo General de Palacio. 1620 


\section{Datos cuantitativos}

\section{Avenidas}

En los 50 años considerados se han detectado avenidas un total de 66 episodios mensuales de avenidas que afectan a cualquiera de los tres tramos de cuenca considerados. De ellos $41 \%$ son de intensidad baja, $36 \%$ media y $23 \%$ alta. Los tramos afectados son de cabecera en el $9 \%$ de los casos, $33 \%$ de tramos medios y 52\% de tramos regionales. Las avenidas ocurren desde octubre a enero y de marzo a mayo, disminuyendo considerablemente entre junio y septiembre. Éstas se concentran en primavera (35\%), invierno (33\%) y otoño (23\%). Las avenidas de primavera se producen en un $66 \%$ en tramos bajos. Las avenidas de invierno y de otoño se producen en todos los tramos de ríos, incluidos los de primer orden. En la distribución estacional se aprecia que hay avenidas en todas las estaciones, especialmente en invierno, hasta la mitad de la decena de los 1570. A continuación, las de invierno y primavera disminuyen y aumentan las de otoño. En la decena de los 90 las avenidas de invierno y primavera aumentan hasta 1594 y decaen rápidamente en estas estaciones hacia el final de la serie, aunque las de otoño se mantienen estables a lo largo de toda la decena.

Figura 3

DISTRIBUCIÓN ESTACIONAL DE PRECIPITACIONES Y AVENIDAS
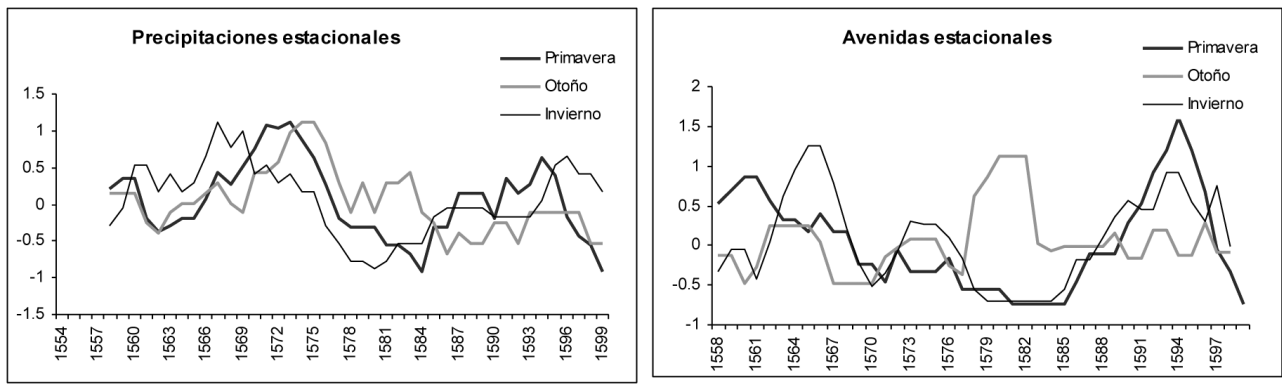

Fuente: Elaboración propia

\section{Precipitaciones}

En el 33\% de los casos registrados las precipitaciones son de intensidad baja, el $48 \%$ media y el $19 \%$ alta y ocurren en invierno (43\%) y (23\%) en primavera y otoño. Entre 1554 y 1573 las precipitaciones son abundantes y se producen en otoño, invierno y primavera, pero en años posteriores las precipitaciones disminuyen mucho en todas las estaciones, especialmente en primavera, sobre todo entre 1574 y 1584 , y se recuperan parcialmente hacia final de siglo. (Figura 4). 


\section{Análisis conjunto}

Los estadísticos descriptivos de las series normales, tabla 1 , muestran que éstas proceden de una distribución normal, ya que los valores de asimetría y curtosis tipificadas son $<2$, por lo que están dentro del rango de los datos esperados de normalidad. Esto asegura la robustez del tratamiento de los datos con métodos paramétricos.

Tabla 1

ESTADÍSTICOS DESCRIPTIVOS DE LAS SERIES ANALIZADAS

\begin{tabular}{|l|c|c|}
\hline & Precipitaciones & Avenidas \\
\hline Promedio & 4.40 & 2.97 \\
\hline Des estándar & 3.38 & 2.82 \\
\hline Asimetría tip. & 1.13 & 1.80 \\
\hline Curtosis tip. & -1.21 & -1.09 \\
\hline
\end{tabular}

Elaboración propia

Las series analizadas no son aleatorias, ya que los estadísticos de Durbin-Watson de precipitaciones y avenidas muestran indicios de correlación serial (precipitación /tiempo $=1.40, \mathrm{p}=0.01$; avenidas/tiempo $=1.23, \mathrm{p}=0.00)$. Los coeficientes de autocorrelación de las series de precipitaciones y de avenidas (autocorrelación residual en Lag1) son 0.83 y 0.82 respectivamente.

La serie normalizada de intensidad/ duración de precipitaciones tiene máximos, con desviaciones respecto al valor normal > 2 en 1556 y 1567 y > 1 en 1570,1572 y 1585 y 1591 . Los mínimos < -1 se dan en 1584, 1588,1589, 1597 y 1599.

La serie normalizada intensidad/ magnitud de las avenidas tiene valores > a 2 en 1563 y $1594,>$ a 1,5 en 1586,1591 y 1597 respecto al valor normal.

Figura 4

SERIES ANUALES DE PRECIPITACIÓN Y AVENIDAS
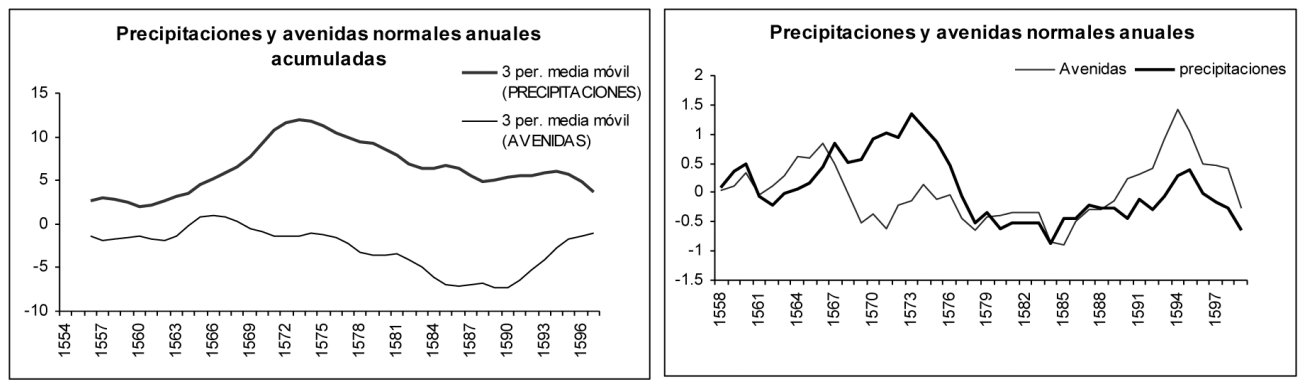

Fuente: Elaboración propia 
Las curvas correspondientes de las respectivas series anuales se aprecian varias fases de máxima y mínima precipitación o avenidas. Las avenidas son frecuentes y de intensidad mantenida entre 1557-1564. Son importantes puntualmente, pero irregulares entre 1591 y 1597 (Figura 5). La curva de valores normales acumulados da resultados similares.

La combinación de las series de avenidas y precipitaciones acumuladas muestra que entre 1552 y 1564 precipitaciones y avenidas tienen trayectorias paralelas entre sí, pero después, entre 1565-1574, el aumento de la intensidad de las precipitaciones no está asociado a avenidas importantes. Tampoco las avenidas de 1585-1599 están relacionadas con un aumento equiparable de las precipitaciones. Las escasas y poco importantes avenidas de 1575-1585 coinciden con una menor intensidad de precipitaciones (Figura 4).

Los grupos de años de similar comportamiento en avenidas y precipitaciones están expresados en el test secuencial de Kendall (Figura 4). En la reprecipitaciones se aprecia un único grupo bien definido, que empieza en 1556-57 y termina hacia 1581, que recoge el período de mayor abundancia de precipitaciones. En la de avenidas se define un primer grupo entre 1558 y 1583 y un segundo grupo a partir de 1590 .

Figura 5

TEST SECUENCIAL DE KENDALL DE AVENIDAS Y PRECIPITACIÓN
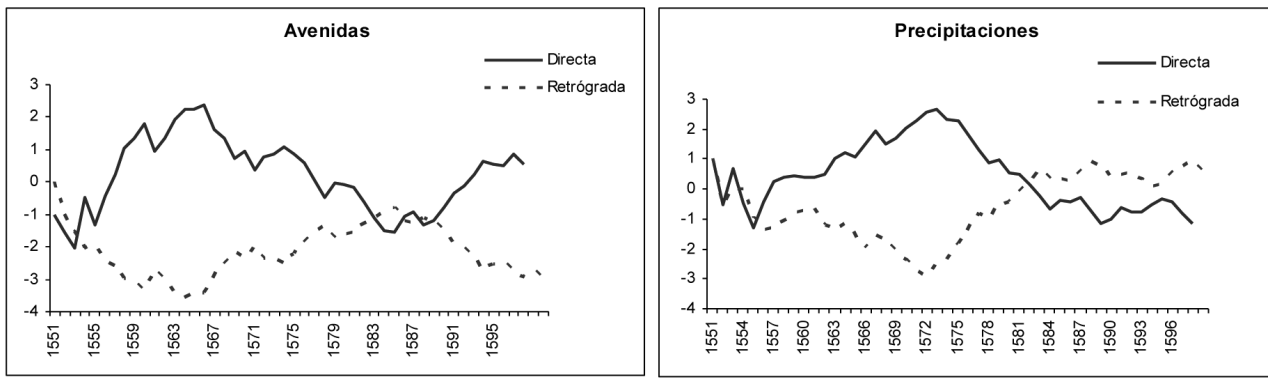

Fuente: Elaboración propia.

\section{INTERPRETACIÓN}

Las avenidas más frecuentes e intensas ocurren en invierno y en los documentos se asocian a la presencia de nieve en las montañas en las que se sitúan las cabeceras fluviales, aunque asimismo hay crecidas de primavera y flash floods estivales, repentinos y efímeros, que son los que generan mayores daños. De acuerdo con Glaser et al. (2010). Estas avenidas podrían deberse, en primer lugar, a la influencia de la precipitación de los frentes atlánticos que alcanzan el centro de la Península Ibérica durante el invierno y la primavera y, en segundo lugar, a una intensa actividad convectiva estival.

La interpretación del desfase entre los años en los que ocurren las precipitaciones y las avenidas de mayor importancia es el problema más importante que plantean los datos presentados. Entre 1556 y 1574 las lluvias son frecuentes a lo largo de todo el año, lo que podría indicar la ocurrencia de un régimen de precipitaciones de intensidad equilibrada con la capacidad del sistema hidrológico de drenaje para canalizar las escorrentías y para favo- 
recer la infiltración del suelo. La alimentación de agua a los ríos sería constante y aunque se producirían con frecuencia caudales de aguas altas, habría pocos desbordamientos.

Desde 1574 hasta el final de las series estudiadas hay una reducción muy importante de las precipitaciones. Todas las informaciones cualitativas recogidas indican que se entra en una fase de sequía prolongada e intensa. Entre 1574 y 1585 la disminución de la precipitación total anual concuerda con la reducción de episodios de precipitación en invierno y primavera, por lo que las avenidas son escasas. Al mismo tiempo, los recursos de agua del suelo tienden a reducirse progresivamente y la vegetación sufre un importante deterioro, tanto en las masas forestales como en las de pastizales. Asimismo, las cosechas anuales de cereal disminuyen.

En la década de los noventa se produce una la recuperación de las precipitaciones, pero lo más llamativo de esa época es la magnitud de las avenidas. Esta situación no parece extraña en la cuenca el Tajo, pues en el análisis de las series contemporáneas de esta cuenca, Potenciano (2004) y Garzón y Potenciano (2007) encuentran que las mayores avenidas se producen en períodos de sequía, tanto en las cuencas mediterráneas como atlánticas. La explicación de ello estaría causada tanto por la intensidad de las precipitaciones como por la menor capacidad de infiltración del sistema hídrico-ambiental. Esta interpretación aplicada a la interpretación de las grandes avenidas del final del siglo XVI encontraría la causa de la disminución de la capacidad de infiltración del suelo en el largo período de sequía de los años previos. Asimismo, la vegetación, similarmente afectada por el déficit hídrico, tendría menos capacidad de intercepción de la lluvia. Debido a ello, la tasa de escorrentía superficial se incrementaría y el caudal de los ríos crecería muy rápidamente y producirían los importantes desbordamientos sobre las llanuras de inundación que indican los datos recogidos. (Figuras 3 y 4).

En todo caso, la recuperación de la lluvia después de un período de sequía que se aprecia en este final del siglo XVI sigue un modelo común en la península Ibérica, muy frecuentemente definido en cuencas ibéricas peninsulares, Olcina (2005), Mateu y Camarasa (2000), y se asocia con frecuencia a la ocurrencia de grandes desastres hidrológicos.

Habría que considerar asimismo la circunstancia de que estos acontecimientos de grandes avenidas ocurren al mismo tiempo que se está produciendo una crisis don temperaturas inferiores a lo normal, con abundancia de episodios de nieve y hielo (Bullón 2008). Por un lado, el deterioro de los suelos castigados por las continuas heladas, que fueron muy intensas durante el período de mayor sequía, disminuiría la capacidad de infiltración de éstos. Por otro, las aportaciones puntuales procedentes de la fusión de la nieve que habría estado retenida en las cabeceras de las cuencas aumentaría la cantidad de agua circulante e incrementaría el caudal de los ríos en mayor proporción de lo que le correspondería debido a las precipitaciones acaecidas. Las mayores avenidas ocurrirían en los años 90 porque esta década coincide con el retorno a la normalidad de las temperaturas, después de 30 años de condiciones frías. El volumen de agua de fusión liberada, al ser canalizado a través de los cauces, aumentaría la magnitud de las avenidas.

Por otro lado, existe una coincidencia general en admitir la importancia de las avenidas de los años finales del siglo XVI. Benito (2006) sitúa el período 1590-1610 dentro de los que tienen crecidas de mayor magnitud dentro de las cuencas atlánticas ibéricas, teniendo en cuenta los últimos 300 años, que, por otra parte, identifica como un período en el que hay 
prolongadas sequías. Por otra parte, Rodrigo et al. (2001) reconocen también la importancia de la década de los 90 en el desarrollo de importantes avenidas en Andalucía.

\section{CONCLUSIONES}

Los datos que se aportan aquí informan sobre un tipo de explotación del territorio ya desaparecido, que es propio de márgenes fluviales caracterizados por la inundación periódica de las riberas y la movilidad del cauce dentro de la llanura de inundación. Esta activa dinámica fluvial genera riesgos importantes en las áreas de influencia de los ríos. Con frecuencia los terrenos ribereños son afectados tanto en los usos del suelo como en los sistemas de propiedad.

Se han detectado tres tipos diferentes de crecidas. Las más habituales son en general beneficiosas y a ellas están adaptados los diferentes usos del suelo y técnicas de acondicionamiento y control de los caudales. Las crecidas extraordinarias producen daños importantes y eventualmente modificaciones del trazado de los cauces. Los flash floods, que ocurren a consecuencia de precipitaciones intensas, son menos conocidos en la definición hidrológica de este sistema fluvial y que tienen importancia en la determinación de los riesgos potenciales de este sector.

Se diferencian tres conjuntos de años con significados hidrológicos diferentes. El primero, desde 1557 a 1575, tiene precipitaciones regulares, que provocan avenidas de menor intensidad. El segundo, desde 1576 a 1584, se caracteriza por precipitaciones y avenidas escasas. El tercero, desde 1585 a 1599, tiene precipitaciones intensas, con avenidas de gran importancia intercaladas entre grandes sequías

Aunque parece comúnmente admitido que las mayores avenidas en las cuencas ibéricas en general y en la cuenca del Tajo en particular se producen en momentos de sequía, la explicación de la gran magnitud de las mismas durante la última década del siglo XVI estaría fundamentada en un régimen de precipitaciones intensas de corta duración y en la reducción por causas diversas de la capacidad de infiltración de los suelos. Asimismo hay que considerar que los cauces podrían haber recibido suplemento de agua adicional procedente de la fusión de la nieve retenida en las cabeceras de la cuenca debido a la progresiva recuperación de las temperaturas que se produce en los años 90, después de 30 años de condiciones térmicas inferiores a la normalidad.

La disminución de las precipitaciones es un rasgo fundamental del comportamiento hidrometeorológico de estos años. El medio natural acusa el menor aporte de humedad, que se manifiesta en el progresivo deterioro de las masas forestales y en el bajo rendimiento de los pastos y cultivos. Esto podría haber influido en la ocurrencia de la profunda crisis que se produce en el sistema económico de base agrícola-ganadera-forestal de la sociedad castellana, que han puesto de manifiesto numerosos historiadores. Estos, que habitualmente han reconocido la posible influencia de las condiciones meteorológicas, no han podido llegar a conocer con la claridad con que se demuestra en este trabajo la importancia de la disminución progresiva de las precipitaciones que se produjo.

El análisis de los datos realizado aporta nuevas evidencias sobre las características hidrológicas y meteorológicas de la segunda mitad del siglo XVI y permite conocer con detalle la secuencia temporal de los principales acontecimientos ocurridos. El análisis de alta resolu- 
ción que se ha realizado ha permitido conocer aspectos muy significativos y desconocidos de uno de los períodos de mayor interés en la evolución histórica del medio natural del centro peninsular ibérico.

Las extracciones de áridos y las regulaciones de los caudales contemporáneas han alterado la dinámica fluvial, de modo que ya no existe la movilidad de los cauces que tan claramente reflejan los documentos históricos consultados. La enorme variación que se ha producido en el comportamiento actual de los ríos en comparación con la situación histórica no está motivada por el cambio en los parámetros meteorológicos sino por la regulación de los caudales, la detracción de agua para abastecimiento y la excesiva extracción de materiales aluviales en las llanuras de inundación. La consecuencia de todo ello ha sido la degradación generalizada del sistema fluvial y la pérdida de gran parte de la capacidad autorreguladora y de autocontrol de avenidas de los cauces.

La dinámica fluvial histórica analizada es la que corresponde a unas condiciones naturales con poca o nula intervención humana, que se recuperaría en la actualidad si el drenaje se regenerara. La inundación anual de las riberas tenía más efectos beneficiosos que perjudiciales, generaba riqueza y era fuente de una gran biodiversidad. La movilidad de los cauces en la llanura de inundación era una garantía de mantenimiento de la llanura aluvial, de redistribución de la carga sedimentaria y de contención de las avenidas. En la situación actual el sistema fluvial tiene limitadas posibilidades de reacción ante la eventualidad de un aumento extraordinario del volumen de agua circulante similar al de las mayores avenidas históricas detectadas.

Los paisajes históricos eminentemente agrarios basados en una la explotación de las riberas adaptada a los ritmos anuales e interanuales de los ríos han dado paso a paisajes urbanos e industriales en los que aguas y sedimentos han cambiado su papel de agentes fundamentales de la organización del territorio en meros objetos de uso y abuso.

\section{BIBLIOGRAFÍA}

ALONSO, A. y GARZÓN, G. (1997): «Efectos sedimentarios de un río fuertemente antropizado: el Jarama», Cuadernos de Geología Ibérica n ${ }^{\circ} 22$, pp. 265-285.

ALONSO, A., FRANCO, F. GARCÍA ANTÓN, M., GARZÓN, G., GÓMEZ MANZANEQUE, F., MORLA, C., SÁNCHEZ HERNANDO, L. J. (1998): «Reconstrucción morfosedimentaria y fitogeográfica del tramo medio del río Jarama (Madrid, España) durante la segunda mitad del Holoceno. Estudio preliminar». Cadernos Lab. Xeolóxigo de Laxe. Coruña, vol 23, pp. 71-88.

ALVAR, A., GARCÍA, E., ZOFIO, J.C., PRIETO, T., ZOZAYA, L. (2005):«Clima, técnica y poderes». Madrid, 1561-1598. Revista de Historia Moderna, n 23, pp. 135-184.

BARRIENDOS, M. COEUR, D. (2004) : «Flood data reconstruction in historical times from non instrumental sources in Spain and France». En BENITO, G. y THORNDYCRAFT: Systemtic, Palaeoflood and Historical Data for the Improvement of Flood Risk Estimation. CSIC. Centro de Ciencias Medioambientales, pp. 29-42.

BENITO, G. (2006): «Riesgos de inundaciones: Tendencias históricas y perspectivas de acuerdo con el cambio climático». Cuaternario y Geomorfología 20 (3-4), pp. 29-44. 
BENITO, G., DÍEZ-HERRERO, A., FERNÁNDEZ DE VILLALTA, M. (2003): «Magnitude and frecuency of flooding in the tagus basin (Centrral Spain) over the last millenium». Climatic Change, 58, pp. 171-192.

BRÁZDIL, R. DOVROVOLNY, P., LUTERBACHER, J., MOBERG, A., PFISTER, CH. WEELER, D. y ZORITA, E. (2010): «European climate of the past 500 years: new challenges for historical climatology». Climatic Change.DOI 10.1007/s10584-009-9783-z.

BULLÓN, T (2008): «Evolución de las temperaturas invernales en la segunda mitad del Siglo XVI en un sector del Sistema Central Español». Boletín de la Asociación de Geógrafos Españoles, 48, pp. 311-325.

BULLÓN, T. (2009): «Precipitaciones y sequías durante los siglos XVI-XVII a partir de datos documentales en un sector del centro de la Península Ibérica». XXI Congreso Nacional de la Asociación de Geógrafos Españoles (AGE). Ciudad Real.

BULLÓN, T. (2010): «La relación de las precipitaciones y sequías con las avenidas fluviales en los agrobiosistemas del conjunto Jarama- Tajo al final del siglo XVI», en Fernández, F, Galán, E., Cañada, R.: Clima, ciudad y ecosistemas. Barcelona, Publicaciones de la Asociación Española de Climatología (AEC), serie A nº 7, pp. 337- 347.

DOMÍNGUEZ-CASTRO, F., SANTISTEBAN, J., BARRIENDOS, M., MEDIAVILLA, R. (2008): «Reconstruction of droughts episodes for central Spain from rogation ceremonies at the Toledo Cathedral from 1506-to 1900: A methodological approach». Global and Planetary Change.

GALLEGO, M. S. y SÁNCHEZ M. A. (2006): «La destrucción ambiental del río Tajo, orígenes, procesos y consecuencias». V Congreso Ibérico sobre gestión y planificación del agua. Faro. Portugal, Comunicaciones. http://www.ualg.pt/5cigpa/es/index.php.

GARZÓN, G. Y POTENCIANO, A. (2007): «Relación entre inundaciones históricas y períodos secos y húmedos. Aproximación a los patrones climáticos del centro-sur de la Península Ibérica por comparación con series temporales de precipitación». Resúmenes XII Reunión Nacional del Cuaternario. AEQUA, Avila.

LHERMITE, J. (2005): «El Pasatiempos. Memorias de un gentilhombre flamenco en la corte de Felipe II y Felipe III». Bruselas. Biblioteca Regia, 690 pp.

OLCINA CANTOS, J. (2007): Prevención de riesgos: cambio climático, sequías e inundaciones. Panel científico-técnico de seguimiento de políticas de agua. Fundación Nueva Cultura del Agua. pp40. http://www.unizar.es/fnca/varios/panel/33.pdf.

LLASAT, M. C., BARRERA, A., BARRIENDOS, M. (2004): «Analysis of climatic condition associated with historical floods». En: BENITO G Y. THORNDYCRAFT, V.R: Systematic, Palaeoflood and Historical Data for the Improvement of Flood Risk Estimation. CSIC. Centro de Ciencias Medioambientales, pp. 85-93 pp.

MATEU, J. CAMARASA, A. (2000): «Las inundaciones en España en los últimos 20 años. Una perspectiva geográfica». Serie Geográfica, 9, pp. 11-15.

MOLINA, P. y BERROCAL, A.( 2006): «Los efectos ambientales de la regulación de los cursos de la cabecera de la cuenca del Tajo: la reducción de los bosques aluviales del Tajo-Jarama». III Congreso de Ingeniería Civil, Territorio y Medio Ambiente. Zaragoza. http://www.ciccp.es/biblio digital/Icitema III/congreso/pdf/030502.pdf. 
PONTENCIANO DE LAS HERAS, A. (2004): Inundaciones históricas en el centro-sur de la península ibérica. Condicionantes geomorfológicos y climáticos. Universidad Complutense de Madrid. Tesis doctoral.

PFISTER, CH. (2020): «The vulnerability of past societies to climatic variation: a new focus for historical climatology in the twenty-first century». Climatic change, 100, pp. 25-31. DOI 10.1007s10584-010-9829-2.

RODRIGO, F.S., ESTEBAN PARRA, M.J., POZO VÁZQUEZ, D. CASTRO DÍEZ, Y. (2001): «A reconstruction of the winter North Atlantic Oscillation index back to A. D. 1501 using documentary data in Southern Spain». J. Geophys. Res (Atmospheres), 106, pp. 14805-14818.

VIZCAÍNO, P., MAGDALENA, F., SEVES, S. MERINO, S., GONZÁLEZ DEL TÁNAGO, M. ( 2003): «Los cambios geomorfológicos del río Jarama como base para su restauración». Limnetica 22 (2-4) pp. 1-8 pp.

\section{RELACIÓN DE ARCHIVOS Y FUENTES CONSULTADAS}

ARCHIVO GENERAL DE SIMANCAS. Casas y Sitios Reales. Legajos: 247, 251, 252, 253, 254, 257, 258, 259, 260, 261, 267, 268, 280, 281, 291, 299, 302, 302.

ARCHIVO GENERAL DE PALACIO. Administraciones Patrimoniales Patronato Aranjuez, cajas 265,290. Patronato San Lorenzo, cajas 3, 6, 11.

ARCHIVO HISTÓRICO NACIONAL (SECC. NOBLEZA): Osuna: 2381, 2384, 2385, 2398, 3151 .

Biblioteca del Real Monasterio de San Lorenzo del Escorial. Libros de actos capitulares. Siglo XVI.

ARCHIVO DE VILLA. MADRID. Libros de actas. Rollo 405/87 números 22, 23, 24.

ARCHIVO REGIONAL DE MADRID. TORRELAGUNA. Libro de acuerdos 906650.

BIBLIOTECA NACIONAL DE ESPAÑA. Manuscritos 9937, 10857, 11561, 11157, 13374

BRITISH LIBRARY. Manuscritos: ADD 28,350, ADD 28,354. 